\title{
ACOSO ESCOLAR EN ESTUDIANTES DE SECUNDARIA: CONSUMO DE ALCOHOL, PERCEPCIÓN DE SALUD Y CALIDAD DE LAS RELACIONES FAMILIARES EN AGRESORES Y/O VÍCTIMAS
}

\author{
BULLYING IN SECONDARY SCHOOL STUDENTS: ALCOHOL USE, PERCEPTION OF \\ HEALTH AND THE QUALITY OF FAMILY RELATIONSHIPS AMONG BULLIES AND/OR \\ THEIR VICTIMS \\ Josefina Patiño-Masó, Maria-Eugenia Gras, Anna Salamó, Montserrat Arboix y Sílvia \\ Font-Mayolas \\ Universidad de Girona (España)
}

\begin{abstract}
El acoso escolar es uno de los más preocupantes problemas de comportamiento en los adolescentes y en todos los centros educativos. Objetivos. Analizar la prevalencia de diversas conductas de acoso escolar en estudiantes de secundaria, como víctimas y como agresores y su relación con el uso de alcohol, el estado de salud y la calidad de las relaciones familiares. Comparar la prevalencia de estas conductas como agresor, entre los adolescentes que las han sufrido y los que no. Método. Estudio comparativo descriptivo transversal. Participaron 468 escolares, edad media $=12.81$ años $(D T=0.75), 49.4 \%$ mujeres. Resultados. Un $36.2 \%$ de los participantes sufrieron o practicaron respectivamente, al menos una, de las conductas analizadas. Las más habituales fueron los insultos y/o las burlas. Se halló que era más frecuente ser agresor si se había sido víctima de acoso escolar o se había probado el alcohol. Las conductas de bullying se asociaron a un peor estado de salud percibido y a una mala calidad de las relaciones familiares. Conclusión. Las acciones preventivas frente al acoso escolar en la infancia y adolescencia deben trabajarse en la familia, en toda la etapa escolar y en la comunidad de forma precoz, sistemática, integrada y gradual.
\end{abstract}

Palabras clave: acoso escolar, educación secundaria, alcohol, estado de salud, relaciones familiares.

Bullying is one of the most worrisome problems among school adolescents. Objective. This paper analyzes the prevalence of several bullying behaviors among adolescent victims and perpetrators in secondary school as well the relationship of those behaviors with alcohol use, perceived health status and the quality of family relationships. It compares the prevalence of these behaviors perpetrators who have been victims of bullying and those who have not. Method. Cross sectional descriptive comparative study. The participants were 468 students whose average age was 12.81 (SD = 0.75) and of whom $49.4 \%$ were female. Results. Among the participants $36 \%$ have been either a victim or a perpetrator of at least one of the bullying behaviors. The most frequent were insults/taunts. Victims of bullying and those who have tried alcohol are more likely to be perpetrators. In general, bullying behaviors are associated with lower perceived health status and poorer quality of family relationships. Conclusion. We consider contextualizing interventions to prevent bullying all stages of development and to extend our focus beyond the various

Josefina Patiño-Masó, http://orcid.org/0000-0002-2322-2437, Instituto de Investigación y Calidad de Vida. Universidad de Girona, Girona. España, correspondencia a josefina.patino@udg.edu.

Maria-Eugenia Gras, http://orcid.org/0000-0003-4765-0871, Instituto de Investigación y Calidad de Vida. Universidad de Girona, Girona. España.

Anna Salamó Avellaneda, http://orcid.org/0000-0002-7019-7967, Instituto de Investigación y Calidad de Vida. Universidad de Girona, Girona. España.

Montserrat Arboix Perejamo, http://orcid.org/0000-0002-5092-7467, Escuela Municipal del Trabajo de Granollers, Barcelona. España.

Sílvia Font-Mayolas, http://orcid.org/0000-0002-4216-3604, Instituto de Investigación y Calidad de Vida. Universidad de Girona, Girona. España. 
scenarios in which children and/or adolescents participate, in order to involve families, teaching teams, students themselves and of course the community at large.

Keywords: bullying, secondary education, alcohol, health status, family relations.

El acoso escolar o bullying es uno de los problemas más preocupantes en los centros educativos (Chester et al., 2015; Falla \& Ortega, 2019; Herrera-López et al., 2017). Se refiere al conjunto de conductas agresivas que una persona o un grupo de personas dirige contra un igual de forma hostil, intencionada, repetitiva, durante un tiempo prolongado, con abuso de poder y voluntad de dañar (Garaigordobil et al., 2017; Olweus, 2013). Se pueden diferenciar diversos tipos de conducta hostigadora: agresiones verbales, físicas, psicológicas-sociales y/o sexuales (Medina \& Reverte, 2019; Marín-Martínez \& Reidl, 2013). Se incluye además el cyberbullying que consiste en el uso de las tecnologías de la Información y Comunicación (TIC) para hostigar y/o acosar a los compañeros (Garaigordobil et al., 2017).

La prevalencia del acoso escolar puede variar en función del contexto (Menesini \& Salvimalli, 2017). Los resultados de un estudio basado en los datos recogidos por distintas encuestas internacionales sobre la salud y el comportamiento de los escolares, de edades comprendidas entre los 13 y los 15 años de edad, señalaron que la prevalencia de acoso escolar era similar para los chicos (34.8\%) y las chicas (30.4\%). Mientras que ellos experimentaron mayor probabilidad que ellas de sufrir agresiones en forma de ataques físicos (38.6\% vs. $25.9 \%$, respectivamente) y burlas (11.6\% vs. $10.3 \%$, respectivamente), las chicas fueron más propensas que los chicos a experimentar acoso psicológico en forma de exclusión social (6.1\% vs. 4.7\%, respectivamente) (United Nations Educational, Scientifical and Cultural Organization [UNESCO], 2019). En España, algunas investigaciones recientes observan que es en la Educación Secundaria Obligatoria (ESO) donde existe una mayor incidencia de acoso escolar (Giménez et al., 2018; Martínez et al., 2019). Los resultados, de la encuesta realizada a los estudiantes españoles de Educación Secundaria Obligatoria de entre 12 y 16 años, indicaron que un 9.3\% declaró haber sufrido acoso escolar (10.6\% chicas y $8.5 \%$ chicos) (Calmaestra et al., 2016). Otra investigación llevada a cabo en Murcia con una muestra ( $n=2503$ ) de alumnos de ESO de distintos centros educativos ( $48.5 \%$ varones) y de edades comprendidas entre los 11 y los 18 años, observó que un 50\% de los participantes se vieron implicados en conductas de violencia entre iguales (Piñero et al., 2014).

El perfil del adolescente que puede verse envuelto en situaciones de acoso escolar es el de un chico/chica diferente al resto del grupo de iguales, con alguna connotación personal que se percibe como negativa respecto a sus compañeros, con pocas habilidades sociales, que está solo/a o tiene pocos amigos y con dificultad para regular sus emociones (González-Cabrera et al., 2019; Salmon et al., 2018).

En la dinámica de acoso escolar cada protagonista juega un rol diferente entre los que se pueden distinguir: el de acosador (bully o bullie) que ejerce la violencia sobre un igual y puede involucrar a otros pares que participan como espectadores ya sea de forma activa o pasiva; el de víctima (victim) persona de la misma comunidad que puede mostrar algún tipo de inferioridad que le hace susceptible para sufrir este tipo de maltrato y el de acosador-victima (bully-victim) que son aquellos jóvenes que después de haber sido víctimas directas de bullying, acaban convirtiéndose en agresores y además pueden de forma simultánea seguir siendo víctimas de otros iguales (Cho \& Wooldrege, 2016; Musalem \& Castro, 2015; Walters, 2020). En esta misma línea, el trabajo desarrollado por Domínguez et al. (2017) constató que los escolares implicados 
en conductas violentas hacia sus iguales, presentaban mayor predisposición para ejercer acoso escolar.

Gaete et al. (2019) observaron un mayor riesgo en el uso de alcohol entre aquellos adolescentes que se ven envueltos en situaciones de acoso escolar. Otras investigaciones refieren que el consumo de alcohol es más común en el grupo de los acosadores y en el de los acosadoresvíctimas, mientras que, en el grupo de las víctimas dicho consumo puede ser incluso inferior al de los chicos y chicas que no están involucrados en el acoso (Seixas et al., 2013; UNESCO, 2019). Según Livingston et al. (2018) el consumo de drogas en algunos adolescentes constituye una herramienta para mitigar los sentimientos negativos causados por el acoso escolar.

Los problemas psicosomáticos y de salud mental son frecuentes en los adolescentes que son víctimas de acoso escolar. Asimismo, pueden observarse afectaciones en la esfera emocional y/o social. Los acosadores y los acosadores-víctimas pueden experimentar problemas semejantes en distinto grado, además de tener un mayor riesgo para involucrarse en interacciones sociales violentas y de consumo de sustancias que a su vez pueden influenciar negativamente en su estado de salud (Musalem \& Castro, 2015). Los resultados del estudio llevado a cabo por la UNESCO (2019), mostraron que las víctimas de acoso escolar tenían el doble de probabilidades de sentirse solos, tener problemas para conciliar el sueño por la noche y haber considerado el suicido, en comparación con aquellos compañeros que no habían sido intimidados por sus iguales. Asimismo, el estado de salud percibido fue menor entre las víctimas de acoso, comparado con aquellos compañeros que no habían sido intimidados por sus iguales. El porcentaje de escolares que aseguró gozar de una salud excelente fue más elevado entre quienes no se habían visto nunca involucrados en conductas de bullying (39.6\%), seguido por los acosadores (33.8\%), las víctimas de acoso escolar (29.1\%) y los acosadores -víctimas (28\%).

Se han descrito diversos factores de riesgo de ser víctima de acoso escolar entre los que se destacan las prácticas parentales mal adaptativas, la negligencia y/o el abuso (González-Cabrera et al., 2019; Suzet et al., 2013). En un estudio sobre acoso escolar y prácticas educacionales se analizaron diversos estilos parentales y se observó que tanto los agresores como las víctimas de bullying informaban de un estilo educativo autoritario o permisivo por parte de sus progenitores en comparación con el resto de los estudiantes. Los progenitores de agresores y víctimas informaron de mayor nivel de estrés parental y específicamente los progenitores de los agresores de menor nivel de competencia parental (Garaigordobil \& Machimbarrena, 2017). Otra investigación halló que las víctimas de acoso escolar percibían mayor afecto y comunicación con sus padres en comparación a los agresores y a los agresores/víctimas, mientras que estos dos grupos se caracterizaban por sentir rechazo y crítica, sobre todo por parte del padre, y poca comunicación y afecto por parte de la madre (León-del-Barco et al., 2015).

Los objetivos de este trabajo son: estudiar la prevalencia de algunas conductas de acoso escolar (insultos o burlas; golpes, ataques o amenazas y marginación o rechazo) como víctima y como agresor según el sexo; comparar el porcentaje de agresores entre los que han sido víctimas de acoso escolar y los que no lo han sido; estudiar la relación entre las conductas de acoso escolar y el uso de alcohol, el estado de salud percibido y la calidad de las relaciones familiares.

\section{Método}

\section{Diseño y Participantes}


Se llevó a cabo un estudio comparativo descriptivo transversal. La población diana estuvo formada por todos los estudiantes de primero y segundo curso de Educación Secundaria Obligatoria de cuatro institutos públicos de áreas urbanas y rurales de las provincias de Barcelona y Girona (España). Participaron un total de 468 estudiantes (49.4\% chicas), con edades comprendidas entre los 11 y los 15 años; edad media = 12.81 años; $D T=0.75$.

\section{Instrumentos}

Para la recogida de información se utilizó el cuestionario de Factores de Riesgo en Estudiantes de Secundaria (FRESC) con permiso de sus autores (Pérez et al., 2013). Este cuestionario evalúa diversos comportamientos y estilos de vida de los estudiantes de secundaria que están relacionados con la salud. Las variables del cuestionario que se utilizaron en este trabajo son las siguientes:

\section{Demográficas. Edad "años" y sexo "chico", "chica".}

Acoso escolar. Se preguntó con qué frecuencia durante los últimos 12 meses en la escuela o durante el trayecto a la escuela "lo/a habían insultado o se habían burlado de él o ella", "le habían pegado, lo/la habían atacado y/o amenazado", "los/as compañeros/as lo/a habían marginado o rechazado que formara parte del grupo". También por si habían participado en la escuela o durante el trayecto a la escuela con otros compañeros o con su grupo en "insultar o burlarse de otros compañeros/as", "pegar, atacar o amenazar a otros compañeros/as", "marginar o rechazar a otros compañeros/as". En todos los casos, las opciones de respuesta ofrecían cinco alternativas: nunca, 1 vez, 2 veces, 3 veces y 4 o más veces. Los participantes se dividieron en dos categorías, los que nunca habían sufrido o realizado la conducta y los que la habían sufrido o realizado alguna vez. La consistencia interna entre los ítems, medida por el coeficiente alfa de Cronbach es de 0.66 .

Consumo de alcohol. Se utilizaron las preguntas del cuestionario FRESC (Pérez et al., 2013), de forma literal, para identificar a los participantes que habían probado el alcohol alguna vez en la vida de los que no lo habían probado nunca, se preguntó "si habían bebido alguna vez al menos medio vaso de cualquier bebida alcohólica". Las opciones de respuesta fueron: nunca, alguna vez en la vida, en los últimos doce meses, en los últimos seis meses, en los últimos 30 días). Los participantes se dividieron en dos categorías, los que nunca habían probado el alcohol y los que sí lo habían hecho.

Estado de salud. Se preguntó "cómo diría que era su salud en general", con cinco posibles respuestas: excelente, muy buena, buena, regular, mala. Los participantes se dividieron en tres categorías según su percepción de salud: excelente o muy buena, buena y regular o mala.

Calidad de las relaciones familiares percibida. Se preguntó "cómo eran habitualmente las relaciones con su familia", con cinco categorías de respuesta: muy buenas, bastante buenas, regulares, bastante malas y muy malas. Los participantes se dividieron en dos categorías: relaciones familiares buenas o muy buenas y relaciones familiares regulares, bastante malas 0 malas.

\section{Procedimiento}


El presente estudio fue aprobado el 4 de mayo de 2017 por el Comité de Ética y Bioseguridad en la Investigación de la Universidad de Girona. Se informó a los padres desde los propios centros educativos y se recogieron los permisos respectivos por parte de los equipos de dirección de cada instituto. El cuestionario fue distribuido a los alumnos en horario lectivo y se dispuso de un tiempo (30-40 minutos) previamente acordado con los centros escolares para la recogida de los datos, que fue supervisada por dos de las autoras de este trabajo que estuvieron presentes en el aula. Se aseguró que los adolescentes tuvieran privacidad para responder el cuestionario. Se explicó a los escolares el objetivo del estudio y se insistió en el carácter confidencial y anónimo de su participación. Para formar parte de la muestra los adolescentes debían de tener previamente el consentimiento firmado por los padres y acceder voluntariamente a participar en el estudio. Un total de 326 alumnos no entregaron el consentimiento por lo que no pudieron participar en el estudio.

\section{Análisis de datos}

Se utilizó la prueba chi-cuadrado para la comparación entre grupos y el coeficiente phi para el cálculo del tamaño del efecto. Todos los análisis se realizaron con el programa estadístico SPSS, Versión 23.

\section{Resultados}

En total un 36.2\% de los participantes informaron haber sido víctimas de una (22.2\%), dos (9.1\%) o las tres conductas (5\%) de acoso escolar estudiadas. El mismo porcentaje (36.2\%) informó haber sido agresor: un 21.9\% indicó que había realizado a otros una de estas agresiones, un $11.1 \%$ que había realizado dos de ellas y un 3.2\% que había realizado las tres. En la Tabla 1 se presentan los porcentajes de adolescentes que informaron de conductas consideradas de acoso escolar como víctimas y como agresores y según el sexo. Cerca de la cuarta parte de la muestra indicó que se habían burlado de ellos o los habían insultado en la escuela o durante el trayecto a la escuela, casi un $13 \%$ que les habían pegado, atacado o amenazado y casi un $18 \%$ que los compañeros los habían marginado o rechazado. Más chicos que chicas informaron de estas conductas, excepto en el caso de la marginación que fue informada más frecuentemente por las chicas, aunque en ningún caso las diferencias tenían significación estadística. Más de la cuarta parte de los adolescentes informó que se habían burlado o habían insultado a otros compañeros en la escuela o en el trayecto de la escuela, observándose diferencias significativas por sexos: más de tres de cada diez chicos y menos de dos de cada diez chicas informaron de esta conducta, aunque el tamaño del efecto fue débil. Casi el $6 \%$ de los participantes informó que había pegado, atacado o amenazado a otros compañeros en la escuela o en el trayecto a la escuela. El porcentaje de chicos que informó de esta conducta fue el doble del de chicas, y la diferencia tendió a la significación estadística. Más de dos de cada diez estudiantes informaron de haber marginado o rechazado a otros compañeros, sin diferencias significativas según el sexo.

Tabla 1

Adolescentes víctimas y agresores según conducta

$\begin{array}{llll}\text { Chicos } & \text { Chicas } & \text { Total } & X^{2}(1)(p)\end{array}$

Víctima 


\begin{tabular}{|c|c|c|c|c|c|}
\hline $\begin{array}{l}\text { Le han } \\
\text { insultado }\end{array}$ & $27.3 \%(n=65)$ & $22.3 \%(n=52)$ & $24.8 \%(n=117)$ & $1.6(.21)$ & .06 \\
\hline Le han pegado & $14.8 \%(n=35)$ & $10.4 \%(n=24)$ & $12.8 \%(n=59)$ & $2.0(.15)$ & .07 \\
\hline $\begin{array}{l}\text { Le han } \\
\text { marginado }\end{array}$ & $16.9 \%(n=40)$ & $18.8 \%(n=43)$ & $17.8 \%(n=83)$ & $.3(.59)$ & .03 \\
\hline \multicolumn{6}{|c|}{ Agresor } \\
\hline Ha insultado & $31.8 \%(n=76)$ & $19.1 \%(n=44)$ & $25.6 \%(n=120)$ & $9.9(.002)^{* *}$ & .15 \\
\hline Ha pegado & $8.0 \%(n=19)$ & $3.9 \%(n=9)$ & $5.9 \%(n=28)$ & $3.6(.06)$ & .09 \\
\hline Ha marginado & $24.9 \%(n=59)$ & $21.5 \%(n=50)$ & $23.2 \%(n=109)$ & $.8(.38)$ & .04 \\
\hline
\end{tabular}

${ }^{* *} p<.01$.

En todos los casos era más frecuente ser agresor si se había sido víctima de acoso escolar. El 45.2\% de los adolescentes que había sido víctima de insultos o burlas también había insultado o se había burlado de otros compañeros, pero solo el $19.3 \%$ de los que no había sufrido insultos o burlas, lo había hecho. Las diferencias fueron significativas en todos los casos excepto en dos: entre las víctimas de burlas el porcentaje de adolescentes que había marginado a otros fue similar al del grupo que no había sido víctima de burlas y entre las víctimas de agresiones físicas el porcentaje de adolescentes que había marginado a otros, no fue significativamente diferente al del grupo que no había sufrido estas agresiones. No obstante, los tamaños del efecto fueron pequeños (Tabla 2).

Tabla 2

Adolescentes agresores entre quienes han sido víctimas de acoso escolar

Víctima

Agresor

\begin{tabular}{lccc} 
& Ha insultado & Ha pegado & Ha marginado \\
\hline Le han insultado & $45.2 \%(n=52)$ & $10.4 \%(n=12)$ & $27.2 \%(n=31)$ \\
No le han insultado & $19.3 \%(n=68)$ & $4.5 \%(n=16)$ & $21.8 \%(n=77)$ \\
\hline$X^{2}$ & 30.5 & 5.4 & 1.4 \\
$p$ & $<.001^{* * *}$ & $.02^{*}$ & .23 \\
Phi & 0.26 & 0.11 & 0.05 \\
\hline Le han pegado & $44.1 \%(n=26)$ & $17.2 \%(n=10)$ & $27.6 \%(n=16)$ \\
\hline No le han pegado & $23.2 \%(n=94)$ & $4.4 \%(n=18)$ & $22.6 \%(n=92)$ \\
\hline$X^{2}$ & 11.7 & 14.8 & .7 \\
\hline
\end{tabular}




\begin{tabular}{lccc}
\hline$p$ & $.001^{* * *}$ & $<.001^{* * *}$ & .40 \\
Phi & 0.16 & .18 & 0.04 \\
\hline Le han marginado & $39.8 \%(n=33)$ & $14.6 \%(n=12)$ & $37.8(n=31)$ \\
No le han marginado & $22.7 \%(n=86)$ & $4.2 \%(n=16)$ & $20.4 \%(n=78)$ \\
\hline$X^{2}$ & 10.4 & 13.0 & 11.4 \\
$p$ & $.001^{* * *}$ & $<.001^{* * *}$ & $.001^{* * *}$ \\
Phi & 0.15 & 0.17 & 0.16
\end{tabular}

${ }^{*} p<.05 .{ }^{* * *} p<.001$.

En la Tabla 3 se presentan los porcentajes de participantes que habían sido víctimas y agresores según el uso de alcohol. A pesar de que los que no habían probado el alcohol eran con más frecuencia víctimas de acoso escolar que los que lo habían probado, las diferencias no fueron significativas. Se observó una relación significativa entre el uso de alcohol y ser agresor: los que habían probado el alcohol habían sido con más frecuencia agresores en las tres conductas observadas siendo los tamaños del efecto pequeños.

Tabla 3

Adolescentes víctimas y agresores según el tipo de conducta en función del uso de alcohol

\begin{tabular}{|c|c|c|c|c|}
\hline & No ha probado el alcohol & Sí ha probado el alcohol & $X^{2}{ }_{(1)}(p)$ & Phi \\
\hline \multicolumn{5}{|c|}{ Víctima } \\
\hline $\begin{array}{l}\text { Le han } \\
\text { insultado }\end{array}$ & $26.1 \%(n=73)$ & $23.1 \%(n=43)$ & $.5(.47)$ & .03 \\
\hline $\begin{array}{l}\text { Le han } \\
\text { pegado }\end{array}$ & $18.8 \%(n=36)$ & $13.4 \%(n=23)$ & $.02(.89)$ & .01 \\
\hline $\begin{array}{l}\text { Le han } \\
\text { marginado }\end{array}$ & $18.2 \%(n=51)$ & $17.3 \%(n=32)$ & $.06(.80)$ & .01 \\
\hline \multicolumn{5}{|c|}{ Agresor } \\
\hline $\begin{array}{l}\text { Ha } \\
\text { insultado }\end{array}$ & $21.1 \%(n=60)$ & $32.6 \%(n=60)$ & $7.7(.005)^{* *}$ & .13 \\
\hline Ha pegado & $3.2 \%(n=9)$ & $10.3 \%(n=19)$ & $10.1(.001)^{* * *}$ & .15 \\
\hline $\begin{array}{l}\mathrm{Ha} \\
\text { marginado }\end{array}$ & $18.0 \%(n=51)$ & $31.4 \%(n=58)$ & $11.3(.001)^{* * *}$ & .16 \\
\hline
\end{tabular}

El estado de salud percibido se relacionó con ser víctima de acoso escolar o agresor en el caso de dos conductas: burlarse o insultar en la escuela o en el trayecto a la escuela y rechazar o marginar. Los adolescentes que percibieron peor salud, habían sido víctimas o agresores en mayor proporción. No se halló relación entre el estado de salud percibido y la conducta de golpear, atacar o amenazar ni como víctima ni como agresor (Tabla 4). 
Tabla 4

Adolescentes víctimas y acosadores/agresores según el tipo de conducta y la salud percibida

\begin{tabular}{|c|c|c|c|c|c|}
\hline & \multicolumn{5}{|c|}{ Salud percibida } \\
\hline & Regular o mala & Buena & $\begin{array}{c}\text { Muy buena o } \\
\text { excelente }\end{array}$ & $X^{2}(2)(p)$ & Phi \\
\hline \multicolumn{6}{|c|}{ Víctima } \\
\hline $\begin{array}{l}\text { Le han } \\
\text { insultado }\end{array}$ & $52.6 \%(n=10)$ & $38.1 \%(n=40)$ & $19.4 \%(n=67)$ & $23.1(<.001)^{* * *}$ & .22 \\
\hline $\begin{array}{l}\text { Le han } \\
\text { pegado }\end{array}$ & $15.8 \%(n=3)$ & $17.5 \%(n=18)$ & $11 \%(n=38)$ & $3.1(.21)$ & .08 \\
\hline $\begin{array}{l}\text { Le han } \\
\text { marginado }\end{array}$ & $47.4 \%(n=9)$ & $25.2 \%(n=26)$ & $13.7 \%(n=47)$ & $19.2(<.001)^{* * *}$ & .20 \\
\hline \multicolumn{6}{|c|}{ Agresor } \\
\hline $\begin{array}{l}\text { Se ha } \\
\text { burlado }\end{array}$ & $42.1 \%(n=8)$ & $33.3 \%(n=35)$ & $22.4 \%(n=77)$ & $7.9(.02)^{*}$ & .13 \\
\hline Ha pegado & $10.5 \%(n=2)$ & $6.7 \%(n=7)$ & $5.2 \%(n=18)$ & $1.1(.57)$ & .05 \\
\hline $\begin{array}{l}\mathrm{Ha} \\
\text { marginado }\end{array}$ & $42.1 \%(n=8)$ & $29.5 \%(n=31)$ & $19.8 \%(n=68)$ & $8.5(.01)^{* *}$ & .14 \\
\hline \multicolumn{6}{|c|}{${ }^{*} p<.05 .{ }^{* *} p<.01 .{ }^{* * *} p<.001}$. \\
\hline \multicolumn{6}{|c|}{$\begin{array}{l}\text { En la Tabla } 5 \text { se presentan los porcentajes de víctimas y agresores según el tipo de conducta } \\
\text { (insultar, pegar o marginar) y la calidad de las relaciones familiares percibida. Se apreció una } \\
\text { relación entre el acoso escolar y la calidad de las relaciones familiares tanto en el caso de las } \\
\text { víctimas como de los agresores. Cuando se informaba de una relación regular o mala con la familia } \\
\text { era más probable ser víctima de acoso escolar o agresor, excepto en el caso de la conducta de } \\
\text { ser golpeado, atacado o amenazado en que la diferencia no tuvo significación estadística en el } \\
\text { caso de las víctimas. }\end{array}$} \\
\hline
\end{tabular}

Tabla 5

Adolescentes víctimas y acosadores/agresores según el tipo de conducta y la calidad de las relaciones familiares percibida

Calidad de las relaciones familiares

\begin{tabular}{|c|c|c|c|c|}
\hline & Regular o mala & Bastante o muy buena & $X^{2}{ }_{(1)}(p)$ & Phi \\
\hline \multicolumn{5}{|c|}{ Víctima } \\
\hline $\begin{array}{l}\text { Le han } \\
\text { insultado }\end{array}$ & $37.1 \%(n=23)$ & $23.2 \%(n=94)$ & $5.6(.02)^{*}$ & .11 \\
\hline
\end{tabular}




\begin{tabular}{|c|c|c|c|}
\hline $\begin{array}{l}\text { Le han } \\
\text { pegado }\end{array}$ & $16.1 \%(n=10)$ & $12.2 \%(n=49)$ & $.7(.38)$ \\
\hline $\begin{array}{l}\text { Le han } \\
\text { marginado }\end{array}$ & $29.0 \%(n=18)$ & $16.2 \%(n=65)$ & $6.0(.01)^{* *}$ \\
\hline
\end{tabular}

\begin{tabular}{|c|c|c|c|c|}
\hline \multicolumn{5}{|c|}{ Agresor } \\
\hline $\begin{array}{l}\text { Se ha } \\
\text { burlado }\end{array}$ & $51.6 \%(n=32)$ & $21.8 \%(n=88)$ & $25.0(<.001)^{* * *}$ & .23 \\
\hline Ha pegado & $14.8 \%(n=9)$ & $4.7 \%(n=19)$ & $9.6(.002)^{* *}$ & .14 \\
\hline $\begin{array}{l}\mathrm{Ha} \\
\text { marginado }\end{array}$ & $41.0 \%(n=25)$ & $20.7 \%(n=84)$ & $12.2(<.001)^{* * *}$ & .16 \\
\hline
\end{tabular}

\section{Discusión}

Más de un tercio de los adolescentes del presente estudio declaró que había sido agresor o víctima de acoso escolar. Una cuarta parte, afirmó haber sido insultado y/o burlado y algo más de uno de cada diez dijo que sus iguales le habían pegado, atacado y/o amenazado. Más de una cuarta parte afirmó haberse burlado de sus iguales y/o haber insultado a otros y casi uno de cada diez reconoció haber pegado, atacado y/o amenazado a sus compañeros. La prevalencia de estas conductas es inferior a la que señala otra reciente investigación con escolares españoles, en la que seis de cada diez habían recibido insultos y cuatro de cada diez se habían sentido amenazados y/o habían sufrido golpes físicos. La mitad señaló que había insultado a sus iguales y cuatro de cada diez reconoció que había amenazado o agredido físicamente a otro chico/a (Calmaestra et al., 2016).

En cambio, los participantes de este trabajo reflejan cifras algo mayores que sus congéneres españoles con respecto a ser víctimas o agresores de exclusión (marginación y/o rechazo) (17.8\% vs. $16.6 \%$ y $23.2 \%$ vs. $22.1 \%$ respectivamente). Cabe señalar que Cataluña registra uno de los porcentajes más elevados de adolescentes que refieren haber vivido situaciones de exclusión (8.8\%) por parte de sus iguales en comparación con el promedio español (8.4\%) (Calmaestra et al., 2016). En este sentido, señalar que entre las consecuencias del rechazo social se encuentran, entre otros, la generación de sentimientos de inferioridad e inseguridad, la disminución de la valoración personal y el pensamiento negativo sobre uno mismo (Becerra et al., 2013).

Los datos obtenidos coinciden, además, con los de otros estudios que observan que el maltrato verbal es la forma de agresión que se da con mayor frecuencia entre iguales (Calmaestra et al., 2016), seguido de la exclusión social y el maltrato físico (Medina \& Reverté, 2019; García et al., 2010). Asimismo, están en la línea de los reportados por otras investigaciones que señalan que los chicos se involucran más que las chicas en las conductas de bullying (Chester et al., 2015; Herrera-López et al., 2017; Pérez et al., 2013).

En este trabajo, no se observan diferencias por sexo entre las víctimas, aunque ellos reconocieron en mayor porcentaje sufrir burlas, insultos y agresiones físicas y ellas conductas de 
marginación. En cambio, mientras Bouffard \& Koeppel (2016) reportaron que la victimización se da mayoritariamente en los chicos, Santamariña-Rubio et al. (2017) observaron que es más común en las chicas. Probablemente las diferentes edades y/o características de los contextos de las muestras de los estudios y/o los instrumentos utilizados para la recogida de información podrían explicar dichas discrepancias.

Los hallazgos de este estudio señalan que ellos significativamente, utilizaron el insulto y la burla con mayor frecuencia que ellas. En cambio, sin diferencias, los chicos utilizaron más la agresión física y las chicas la conducta de marginación. Estos resultados son concordantes con los de otra investigación que encontró diferencias inter-género entre víctimas y agresores en función del tipo de conducta hostigadora sufrida o perpetrada siendo ellos más propensos a experimentar formas de maltrato directo y ellas a involucrarse en formas indirectas (Salmon et al., 2018).

Los resultados obtenidos en este trabajo evidencian una estrecha relación entre ser víctima y agresor. La probabilidad de maltratar a otros iguales aumenta significativamente si se ha sido víctima de bullying. Estos datos están en consonancia con los reportados por otras investigaciones que observaron que las víctimas de bullying, presentan niveles elevados de emocionalidad negativa y pueden reaccionar desarrollando respuestas agresivas parecidas a las que han sufrido (Domínguez et al., 2017; Hemphill et al., 2011; Walters, 2020).

De acuerdo con los resultados encontrados, no se hallan diferencias significativas entre las víctimas que han probado el alcohol y las que no lo han hecho, en cambio, se observa que los agresores han probado el alcohol con mayor frecuencia y la diferencia es significativa en las tres conductas estudiadas. Estos datos están en sintonía con los reportados por Archimi and Kunstche (2014), que identificaron un mayor uso de alcohol en los agresores y agresores/víctimas, pero no en las víctimas. Son concordantes también con los de otra investigación que observó que los agresores que bebían alcohol utilizaban con mayor frecuencia las agresiones verbales, seguidas de la exclusión social y las agresiones físicas (Luk et al., 2012).

Por lo que se refiere al estado de salud percibido por los adolescentes de la muestra (sean víctimas o agresores), se halla relación con el acoso escolar, concretamente en las conductas de: insultar y/o burlar y marginar y/o rechazar, aunque no ocurre lo mismo respecto a las conductas de pegar o golpear/atacar y/o amenazar. Los participantes que percibían peor estado de salud eran con mayor frecuencia las víctimas de acoso escolar y se observó que quien con mayor frecuencia maltrataba a sus iguales también percibía peor estado de salud. Estos resultados son concordantes con los de otras investigaciones que identifican que los adolescentes que se ven envueltos en el acoso escolar refieren peor salud física, mental y/o social que aquellos que no participan en estas dinámicas (Wolke \& Lereya, 2015; UNESCO, 2019).

Se halla relación entre el acoso escolar y la calidad de las relaciones familiares de modo que si se percibe una relación regular o mala, existe mayor probabilidad de practicar bullying a otros, que si se percibe una relación familiar bastante buena o muy buena. Concretamente en las conductas de insultar y/o burlar o marginar y/o rechazar, se observó una mayor probabilidad de ser víctima de acoso escolar si se percibía una relación regular o mala con la familia. En el caso de los agresores, la diferencia fue significativa en las tres conductas estudiadas. Estos resultados están en sintonía con los de otros estudios que relacionaron los factores de riesgo familiar con el desarrollo de conductas de bullying (Alonso-Castillo et al.,2017; González-Cabrera, 2019; Panfile et al., 2017). 
Este trabajo presenta algunas limitaciones por lo que los hallazgos deben interpretarse con cautela. Al ser un estudio descriptivo transversal no permite determinar relaciones causales entre las variables analizadas. Al estudiarse el colectivo de escolares de dos municipios pequeños puede existir sesgo muestral por no representar a la población general adolescente. Aunque se garantizó el anonimato y la confidencialidad de los datos, los resultados pueden verse afectados por el sesgo de deseabilidad social.

\section{Conclusiones}

Se concluye que nuestros resultados añaden evidencia al hecho de que hoy en día, más de un tercio de los escolares se ven implicados en la problemática del acoso escolar. La importancia de este estudio radica además en el análisis de distintos tipos de conducta hostigadora entre iguales lo que permite explorar diferencias inter- género entre víctimas y acosadores. Asimismo, se demuestra que algunos factores contextuales como el clima familiar negativo y/o el acoso por parte de los iguales, pueden derivar en el aumento de la conducta agresiva en el entorno escolar, así como en comportamientos de riesgo social y para la salud como son: el consumo de alcohol y/o la percepción negativa de la salud, aspectos que repercuten en el aumento de la vulnerabilidad de los individuos.

Destacamos que la conducta de marginar y/o rechazar a un igual, la practican en porcentajes similares tanto quienes han recibido insultos y/o burlas, golpes, amenazas y/o ataques como quienes no han sufrido dichos tipos de agresión. Consideramos que estos resultados son relevantes y deben tenerse en cuenta en las intervenciones de prevención del bullying y en aquellas que se lleven a cabo para promocionar la salud de los escolares.

Independientemente de los motivos que pueden influenciar a los adolescentes para consumir alcohol y/u otras drogas, es importante que el acoso escolar sea considerado como un indicador de riesgo para el uso y/o abuso de sustancias.

Por todo ello, es necesario contextualizar las intervenciones preventivas frente al bullying en todas las etapas de desarrollo, en los distintos escenarios donde participan los niños y/o adolescentes, implicando a las familias, a los equipos docentes, a los propios alumnos y por supuesto al entorno comunitario.

Siendo el contexto educativo el lugar donde se generan principalmente las dinámicas de acoso escolar, consideramos necesario focalizar la intervención en acciones de promoción y/o prevención de la salud que ayuden a identificar de forma precoz las conductas de acoso entre iguales y proporcionar a la comunidad educativa (profesores, padres/madres, monitores deportivos, alumnos, etc.) alternativas para poder evitar dichos comportamientos. Los programas preventivos, deben contemplar el seguimiento y la evaluación de la calidad de las relaciones familiares desde la más tierna infancia con el fin de promover cambios en el cuidado de los hijos que potencien un crecimiento y desarrollo saludable. También deben reforzar la educación emocional, la adquisición de habilidades sociales y el aprendizaje de estrategias que favorezcan la resiliencia de los niños, niñas y/o los/las adolescentes. Todo ello, con el fin de revertir de forma positiva en la convivencia de la comunidad educativa.

\section{Conflicto de intereses}

Las autoras no refieren ningún potencial conflicto de interés en relación con este artículo. 


\section{Referencias}

Alonso-Castillo, M. A., Yáñez-Lozano, A., \& Armendáriz-García, N.A. (2017). Funcionalidad familiar y consumo de alcohol en adolescentes de secundaria. Health and Addictions/Salud y Drogas, 17(1), 87-96.

Archimi, A., \& Kuntsche, E. (2014). ¿Do offenders and victims drink for different reasons? Testing mediation of drinking motives in the link between bullying subgroups and alcohol use in adolescence. Addictive Behaviors, 39, 713-716.

Becerra, S., Pía, M., Véjar, M., \& Vidal, N. (2013). Violence and social rejection at school: their effects in indigenous student. Revista Teoria e Práctica da Educaçao, 16(2), 9-18.

Boufard, L. A., \& Koeppel, M. D. H. (2016). Sex differences in the health risk behaviour outcomes of childhood bullying victimization. Victims \& Offenders, 00, 1-22. doi:10/1080/15564886.2015.1118420

Calmaestra, J., Escorial, A., García, P., Del Moral, C., Perazzo, C., \& Ubrich, T. (2016). Yo a eso no juego. Bullying y ciberbullying en la infancia. Madrid, España: Save the Children, España.

Chester, K. L., Callaghan, M., Cosma, A., Donnelly, P., Craig, W., Walsh, S., \& Molcho, M. (2015). Cross-national time trends in bullying victimization in 33 countries among children aged 11, 13 and 15 from 2002 to 2010. European Journal of Public Health, 25(2), 61-64. doi:10.1093/eurpub/ckv029

Cho, S., \& Wooldredge, J. (2016). The link between juvenile offending and victimization: Sources of change over time in bullying victimization risk among South Korean adolescents. Children and Youth Services Review, 71, 119-129. doi: 10.1016/j.childyouth.2016.10.041

Domínguez, J., Álvarez, E., \&Vázquez, E. (2017). Dimensiones predictivas del constructo violencia escolar en la educación secundaria obligatoria. Revista de Investigación Educativa, 35(2), 337-351. doi: 10.6018/rie.35.2.259471

Falla, D., \& Ortega-Ruiz, R. (2019). Los escolares diagnosticados con trastorno del espectro autista y víctimas de acoso escolar: una revisión sistemática. Psicología Educativa, 25(2), 77-9. doi.org/10.5093/psed2019a6

Gaete, J., Tornero, B., Valenzuela, D., Rojas-Barahona, C. A., Salmivalli, C., Valenzuela, E., \& Araya, R. (2017). Substance use among adolescents involved in bullying: A cross - sectional multilevel study. Frontiers in Psychology, 8,1056. doi:10.3389/fpsyg.2017.01056

Garaigordobil, M., \& Machimbarrena, J. M. (2017). Stress, competence, and parental educational styles in victims and aggressors of bullying and cyberbullying. Psicothema, 29(3), 335-340. doi:10.7334/psicothema2016.258

Garaigordobil, M., Martínez-Valderrey, V., \& Machimbarrena, J.M. (2017). Intervención en el bullying y ciberbullying: Evaluación del caso Martín. Revista de Psicología Clínica con Niños y Adolescentes, 4(1), 25-32. 
García, X., Pérez, A., \& Nebot, M. (2010). Factores relacionados con el acoso escolar (bullying) en los adolescentes de Barcelona [Factors related to bullying in adolescents in Barcelona (Spain)]. Gaceta Sanitaria, 24(2), 103-108. doi:10.1016/j.gaceta.2009.09.017

Giménez-Gualdo, A., Arnaiz-Sánchez, P., Cerezo-Ramírez, F., \& Prodócimo, E. (2018). Percepción de docentes y estudiantes sobre el ciberacoso. Estrategias de intervención en Primaria y Secundaria. Comunicar, 56(XXVI), 29-38. doi:10.39216/C56.2018.03

González-Cabrera, J., Tourón, J., Machimbarrena, J.M., León-Mejía, A., \& Gutiérrez-Ortega, M. (2019). Estudio exploratorio sobre acoso escolar en alumnado con altas capacidades: prevalencia y afectación psicológica. Revista de Educacuón, 386,187-214. doi: 10.4438/1988-592X-RE-2019-386-432

Hemphill, S. A, Kotevski, A., Herrenkohl, T. I., Bond, L., Kim, M. J., Toumbourou, J. W., \& Catalano, R. F. (2011). Longitudinal consequences of adolescent bullying perpetration and victimization: a study of students in Victoria, Australia. Crime Behavior Mental Health, 21(2), 107-16. doi:10.1002/cbm.802

Herrera-López, M., Romera, E., \& Ortega-Ruiz, O. (2017). Bullying y ciberbullying en Colombia, concurrencia en adolescentes escolarizados [Bullying and cyberbullying in Colombia; cooccurrence in adolescent schoolchildren]. Revista Latinoamericana de Psicología, 49, 163-172. doi: 10.1016/j.rlp.2016.08.001

León-del-Barco, B., Felipe-Castaño, E., Polo-del-Río, M. I., \& Fajardo-Bullón, F. (2015). Aceptaciónrechazo parental y perfiles de victimización y agresión en situaciones de bullying [Parental acceptance-rejection and profiles of victimization and aggression in bullying situation]. Anales de Psicología, 31(2), 600-606. doi:10.6018/analesps.31.2.156391

Livingston, J.A., Derrick, J.L., Wang, W., Testa, M., Nickerson, A.B., Espelage, D.L., \& Miller, K.E. (2018). Proximal associations among bullying, mood and substance use: a daily report study. Journal of Child and Famuly Sudies, 28(9), 2558-2571. doi:10.10007/s10826-018$1109-1$

Luk, W. L., Wang, J., \& Simons-Morton, B. G. (2012). The co-occurrence of substance use and bullying behaviors among U.S. adolescents: understanding demographics characteristics and social influences. Journal of Adolescence, 35, 1351-1360. doi: 10.1016/j.adolscence.2012.05.003

Marín-Martínez, A., \& Reidl, L. M. (2013). Validación psicométrica del cuestionario "así nos llevamos en la escuela" para evaluar el hostigamiento escolar (bullying) en primarias [Bullying instrument assessment in elementary school]. Revista Mexicana de Investigación Educativa, 18(56), 11-36.

Martínez, I., Gómez, E.I., Goig, R. (2019). El acoso escolar en educación secundaria: prevalencia y abordaje a través de un estudio de caso. Revista Internacional de Trabajo Social y Ciencias Sociales, 17, 71-91. doi: 10.5944/comunitaria.17.4 
Medina, J.A., \& Reverte, M-J. (2019). Violencia escolar, rasgos de prevalencia en la victimización individual y grupal de la Educación Obligatoria en España. Revista de Estudios y Experiencias en Educación, 18(37), 97-110. doi: 10.21703/rexe.20191837medina9

Menesini, E., \& Salvimalli, C. (2017). Bullying in schools: the state of knowledge and effective interventions. Psychology, Health and Medicine, 22(S1), 240-253. doi:10.1080/13548506.2017.1279740

Musalem, R., \& Castro, P. (2015). Qué se sabe de bullying [What is known about bullying]. Revista Médica Clínica Las Condes, 26(1), 14-23. doi:10.1016/j.rmclc.2014.12.002

Olweus, D. (1993). Bullying at school: What we know and what we can do. Malden MA: Blackwell Publishing.

Panfile, T., Laible, D., \& Angustine, M. (2017). The influences of parent and peer attachment on bullying. Journal of Child \& Family Studies, 26(5), 1388-1397. doi:10.1007/s10826-017$\underline{0663-2}$

Pérez, A., García-Continente, X., \& Grup Col.laborador enquesta FRESC 2012. (2013). Informe FRESC 2012:25 anys d'enquestes a adolescents escolaritzats de Barcelona. Barcelona: Agència de Salut Pública de Barcelona.

Piñero, E., Arense, J.J., López, J.J., \& Torres, A.M. (2014). Incidencia de la violencia y victimización escolar en estudiantes de educación secundaria obligatoria en la Región de Murcia. Revista de Investigación Educativa, 32(1), 223-241. doi: 10.6018/rie.32.1.154251

Salmon, S., Turner, S., Taillieu, T., Fortier, J., \& Afifi, T. O. (2018). Bullying, victimization, experiences among middle and high school adolescents: Traditional bullying, discriminatory harassment and cybervictimisation. Journal of Adolescence, 63, 29-40. doi: 10.1016/j.adolescence.2017.12.005

Santamariña-Rubio, E., Serral, G., Pérez, C., Ariza, C., \& Grup Col.laborador enquesta FRESC 2016. (2017). La salut i els seus determinants en l'alumnat adolescent de Barcelona. Enquesta FRESC 2016. Barcelona: Agència de Salut Pública de Barcelona.

Seixas, S. R., Pinto, J., \& Nicolas-Fisher, G. (2013). Bullies, victims and bully-victims. Impact on health profile. Educaçao, Sociedade \& Culturas, 38, 53-75.

Suzet, T. L., Muthanna, S., \& Dieter, W. (2013). Parenting behaviour and the risk of becoming a victim and a bully/victim: A meta-analysis study. Child Abuse \& Neglect, 37(12), 10911108. doi: 10.1016/i.chiabu.2013.03.001

United Nations Educational, Scientifical and Cultural Organization (UNESCO). (2019). School violence and bullying: global status and trends, drivers and consequences. France: UNESCO.

Walters, G.D. (2020). School-age victimization and perpetration: A Meta-Analysis of prospective studies and research. Trauma, Violence \& Abuse, X(XX),1-11 doi:10.1177/1524838020906513 
Acoso escolar en estudiantes de secundaria: consumo de alcohol, percepción de salud y calidad de las relaciones familiares...

Wolke, D., \& Lereya, S. T. (2015) Long-term effects of bullying. Archives of Disease in Childhood, 100, 879-885. doi:10.1136/archdischild-2014-306667 